\title{
Communications
}

\section{Dynamics of Photo-excited 9-dicanovinyljulolidine in Poly(ethylene glycol)-poly(acrylic acid) Blends}

\author{
Ah-Young Jee and Minyung Lee* \\ Department of Chemistry and Nanoscience, Ewha Womans University, Seoul 120-750, Korea. *E-mail: mylee@ewha.ac.kr \\ Received August 25, 2011, Accepted September 9, 2011
}

Key Words : Fluorescence, Modulus, PEO, PAA, DCVJ

Complex modulus of materials consists of loss and storage parts. Molecular motions in the condensed phase are governed by loss modulus in the viscosity-controlled regime and by storage modulus in the modulus-controlled regime. There are numerous reports on viscosity dependence of photoisomerizion processes, while elasticity dependence has drawn relatively less attention. ${ }^{1,2}$ In this work, we investigated the rigidity influence on the excited-state dynamics of 9-dicyanovinyljulolidine (DCVJ) in poly(ethylene glycol) (PEG)-poly(acrylic acid) (PAA) blends. DCVJ, undergoing an internal twisting motion in the excited state, has been used as a medium rigidity probe. ${ }^{1}$ Figure 1 depicts the molecular structure of DCVJ in which the internal twisting in the excited state occurs around the double bond between julolidine and dicyano moieties. ${ }^{1}$ The excited-state rate process of DCVJ has been known to be barrierless with a flat potential. ${ }^{3}$ PEG-PAA blends form favorable intermolecular complexes. ${ }^{4}$ By changing the weight ratio of PEG/PAA, it is expected that the blends provide a broad range of the Young's modulus of medium. ${ }^{5}$

PEG (MW 2,000) and PAA (MW 240,000) were bought from Sigma-Aldrich and used as received for polymer blends. Each polymer blend film containing DCVJ (SigmaAldrich) was made by spin casting on a cleaned cover glass. The water solvents left in the film were eliminated in an oven at $90{ }^{\circ} \mathrm{C}$ for $24 \mathrm{~h}$. The thickness of polymer films is about $1.2 \mu \mathrm{m}$ and the concentration of DCVJ was ca. $2 \mu \mathrm{M}$. The nanoindentation measurements were performed with an atomic force microscope (Park Systems, XE-100). A silicon tip (910-NCHR, Nanosensor) was used to obtain the loading curves which have information on the Young's modulus. The force constant of the cantilever is $42 \mathrm{~N} / \mathrm{m}$ with the

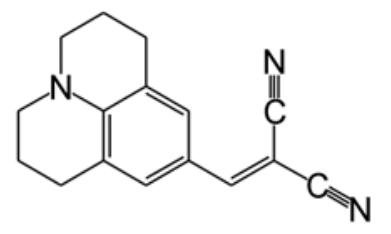

Figure 1. The molecular structure of DCVJ. resonance frequency of $330 \mathrm{kHz}$. For a thin film supported on a hard substance, the Dimitriadis model rather than the original Hertz model has a better description to fit the forceindentation curve: ${ }^{6,7}$

$$
\begin{aligned}
F(\delta) & =\frac{E}{1-v^{2}} \frac{\tan \alpha}{\sqrt{2}} \delta^{2}\left[1+1.133 \chi+1.283 \chi^{2}+0.769 \chi^{3}+0.0975 \chi^{4}\right] \\
\chi & =\sqrt{R \delta} / h
\end{aligned}
$$

where $F$ is the loading force, $E$ is the Young's modulus, $\delta$ is the indentation depth, $\alpha$ is the tip half-angle, $R$ is the tip radius, and $h$ is the sample thickness. The fluorescence lifetimes of DCVJ were measured by time-correlated single photon counting (TCSPC). A picosecond diode laser (Picoquant, PDL-800B) operating at a wavelength of $467 \mathrm{~nm}$ at $10 \mathrm{MHz}$ was used to excite samples mounted on an inverted confocal microscope (Nikon, TE2000-S). The fluorescence photons were collected and processed with a fast TCSPC board (B\&H, SPC 830), and fluorescence lifetimes were extracted from the measured decay curves by a nonlinear least square fit with deconvolution.

According to Eq. (1), the loading force varies nonlinearly with the indentation depth. Figure 2(a) shows the AFM nanoindentation results of PEG-PAA blend samples, together with the fit by the Dimitriadis model. The tip was specified as having an apex radius of $10 \mathrm{~nm}$ and the half angle of $20^{\circ}$. The $v$ value of 0.3 was used for PEG-PAA blends. The sample thickness measured by AFM independently was used for the fit. In the force range from 0 to $6 \mu \mathrm{N}$, the obtained E values of PEG-PAA blends were $0.03,0.08$, $0.13,0.41,0.95,1.87,2.97,3.50$, and $4.24 \mathrm{GPa}$ for $0,10,25$, $40,55,70,85,95$, and 100 wt \% of PAA in PEG. Figure 2(b) shows the fluorescence decays of 4-DASPI in PEG-PAA blends. None of these data show a single exponential, due to the structural heterogeneities of the medium. Therefore, the decay data were fitted to a double exponential form and the average lifetimes were obtained using $\langle\tau\rangle=\mathrm{a}_{\mathrm{i}} \tau_{\mathrm{i}} / \Sigma \mathrm{a}_{\mathrm{i}}$, where $\mathrm{a}_{\mathrm{i}}$ and $\tau_{\mathrm{i}}$ are the amplitude and lifetime components, respectively. The obtained $\langle\tau\rangle$ values were $0.27,0.33,0.47$, $0.62,0.89,1.25,1.44,1.57$, and 1.67 ns for $0,10,25,40,55$, $70,85,95$, and $100 \mathrm{wt} \%$ of PAA in PEG. 
(a)

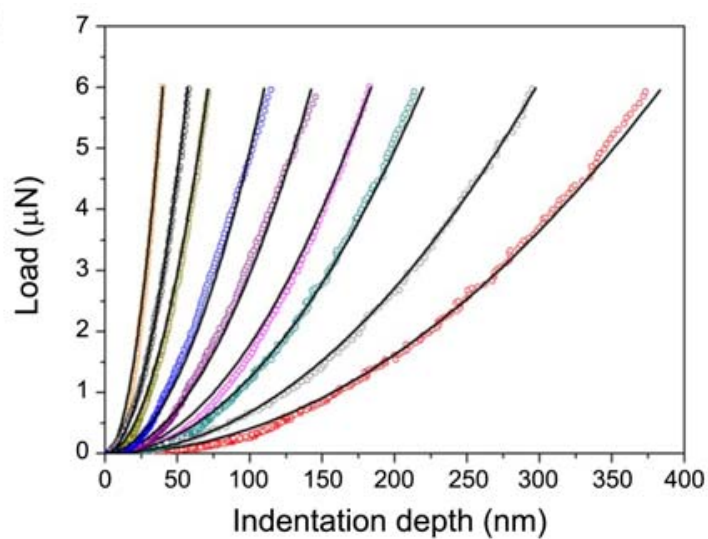

(b)

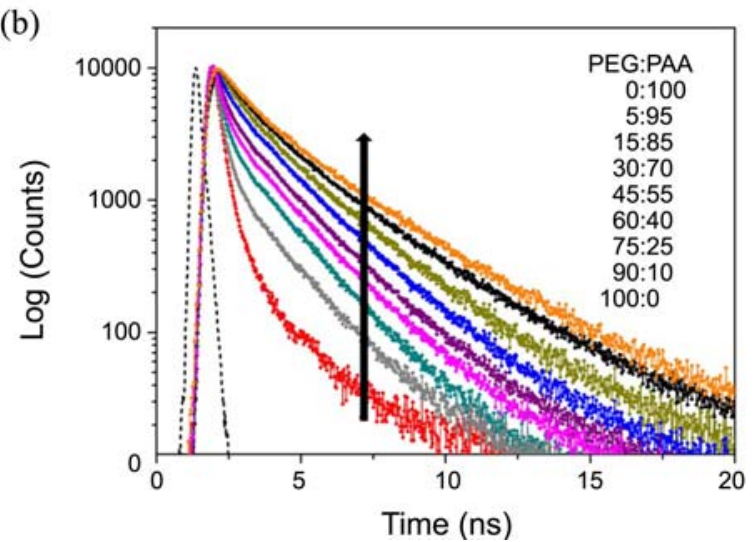

Figure 2. (a) The AFM loading data of PEO-PAA blends and the solid lines from the Dimitriadis model. (b) The fluorescence decay curves of DCVJ in PEG-PAA blends.

The average fluorescence lifetime is associated with the rate constant of the twisting motion by

$$
\langle\tau\rangle=\frac{1}{k_{r}+\sum k_{n r}}
$$

where $k_{r}$ is the radiative rate constant, and $k_{n r}$ is the sum of the nonradiative rate constants responsible for internal conversion, intersystem crossing, and twisting. If the twisting motion dominates other nonradiative decay processes, then the rate constant for the twisting motion, $k_{i r}$, is given as ${ }^{1,5}$

$$
k_{i r}=\frac{1}{\langle\tau\rangle}-k_{r}
$$

The $k_{i r}$ of DCVJ was calculated using the radiative rate constant of $2.8 \times 10^{8} \mathrm{~s}^{-1}{ }^{9}$. Figure 3 shows the twisting rate constant as a function of the Young's modulus of the PEGPAA blends. The fit is given by fractional dependence of the lifetime on the elastic modulus, $k_{i r}=\alpha E^{-\beta}$, with the $\alpha$ and $\beta$ values of $0.71\left(\mathrm{~ns}^{-1}\right)$ and 0.43 , respectively. The fit is satisfactory, indicating that the Young's modulus of matrix governs the excited state dynamics of DCVJ in the PEGPAA polymer blends. The fluorescence lifetimes of DCVJ have been measured in various polymers and were related to

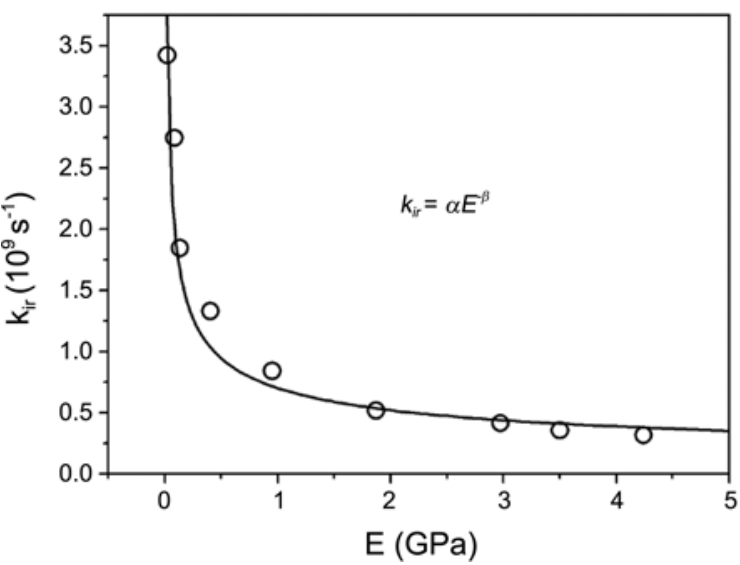

Figure 3. The rate constant for the internal rotation of DCVJ vs. the Young's modulus of polymer blends.

the Young's moduli which were taken in literature. ${ }^{1}$ However, it is more desirable to measure directly the Young's modulus of matrix in which the photoisomerizing dye is embedded. In this work, the $E$ values of the matrices were measured by AFM and used to explain the dynamics of DCVJ in the medium. Another advantage using a blend is that the Young's modulus can be tuned continuously by varying the relative ratio of the polymers.

In conclusion, we have investigated the excited-state motion of DCVJ in the polymer blends of poly(ethylene glycol) (PEO) and poly(acrylic acid) (PAA) that form a favorable interpolymer complex through hydrogen bonding. The Young's modulus of PEG-PAA blends were measured by AFM nanoindentation and the fluorescence lifetime of the dye by TCSPC. The fluorescence lifetime of DCVJ was strongly correlated to the Young's modulus of medium. We have found that the rate constant for the internal motion of DCVJ exhibits fractional dependence on the elasticity.

Acknowledgments. This work was supported by Basic Science Research Program (2010-0541-1) and Acceleration Research Program through the National Research Foundation of Korea.

\section{References}

1. Jee, A.-Y.; Bae, E.; Lee, M. J. Phys. Chem. B 2009, 113, 16508.

2. Jee, A.-Y.; Lee, M. Bull. Korean Chem. Soc. 2010, 31, 1829.

3. Allen, B. D.; Benniston, A. C.; Harriman, A.; Rostron, S. A.; Yu, C. Phys. Chem. Chem. Phys. 2005, 7, 3035.

4. Miyoshi, T.; Takegoshi, K.; Terao, T. Macromolecules 1999, 32, 8914.

5. Jee, A.-Y.; Lee, M. ChemPhysChem 2010, 11, 793.

6. Hertz, H. J. Reine Angew. Math. 1881, 92, 156.

7. Dimitriadis, E. K.; Horkay, F.; Marsca, J.; Kachar, B.; Chadwick, R. S. Biophys. J. 2002, 82, 2798.

8. Nielson, L. E. Mechanical Properties of Polymers; Reinhold: New York, 1967.

9. Strickler, S. J.; Berg, R. A. J. Chem. Phys. 1962, 37, 814. 